Revista Brasileira de

Engenharia Agrícola e Ambiental

v.16, n.10, p.1115-1120, 2012

Campina Grande, PB, UAEA/UFCG - http://www.agriambi.com.br

agriambi Protocolo 253.11 - 28/10/2011 • Aprovado em 16/07/2012

\title{
Modelos matemáticos para a secagem intermitente de arroz em casca
}

\author{
Volnei L. Meneghetti ${ }^{1}$, Elvio Aosani ${ }^{1}$, Jeferson C. da Rocha ${ }^{1}$, \\ Maurício de Oliveira ${ }^{1}$, Moacir C. Elias ${ }^{1} \&$ Ricardo S. Pohndorf ${ }^{1}$
}

\begin{abstract}
RESU M O
Propôs-se, com este trabalho, ajustar modelos matemáticos aos valores experimentais em função do teor de água e estudar os efeitos das relações de intermitência na secagem de grãos de arroz na eficiência da operação. Utilizaram-se grãos de arroz com casca produzidos na Região Sul do Rio Grande do Sul. As amostras com teor de água próximo a $20 \%$ b.u. foram secadas sob três condições de relação de intermitência: 1:1, 1:2 e 1:4; em todas as condicões foram utilizados os mesmos manejos térmicos em temperaturas graduais crescentes do ar: $70,90,110 \pm 5^{\circ} \mathrm{C}$, respectivamente, na 1 a e na 2 a e a partir da 3a hora. Para os ajustes dos modelos matemáticos foi realizada análise de regressão não-linear pelo método Simplex e Q uasi-N ew ton, cujos valores são os parâmetros dos modelos estimados em função da temperatura do ar de secagem. Comparando-se os coeficientes de determinação ajustados $\left(R^{2}{ }_{\text {ajustado }}\right), 0$ erro médio relativo (P) e estimado (SE) e a distribuição de resíduos, os resultados obtidos permitem concluir que: a relação de intermitência altera o tempo de operação e a taxa horária de secagem dos grãos de arroz e, ainda, o modelo de Midilli foi o que melhor representou a secagem intermitente do arroz em casca.
\end{abstract}

Palavras-chave: secador, O ryza sativa, curvas de secagem

\section{Mathematical models for intermittent drying of rice}

\begin{abstract}
This study aimed to adjust mathematical models to experimental values in function of the water content and study the effects of intermittent relations in drying of paddy grains in the efficiency of operation. Paddy grains produced in the southern region of Rio Grande do Sul were used. The samples with approximately $20 \%$ water content were dried under three intermittent relation conditions: 1:1, 1:2, 1:4. In all these conditions the same thermal procedures were utilized, gradual increase in air temperature 70 , $90,110 \pm 5 \stackrel{\circ}{C}$ respectively, in the $1^{\text {st }}, 2^{\text {nd }}$ and $3^{\text {rd }}$ hour. For the adjustments of the mathematical models, a non linear regression analysis through the Simplex and Q uasi-N ew ton methods was carried out. The values of the parameters of the models being estimated in function of the temperature of the drying air. $A$ comparison was carried out of the adjusted coefficient of determination $\left(\mathrm{R}^{2}{ }_{\text {adiusted }}\right)$, the relative mean error (P), the estimated mean error (SE) and the distribution of residues. The results showed that the intermittent relation modifies the operating time and the hourly rate of drying of the rice grains and the Midilli model was the one which best represented the intermittent drying of paddy.
\end{abstract}

Key words: dryer, O ryza sativa, curves of drying 


\section{INTRODUÇÃO}

A água é o componente principal que controla a atividade biológica dos produtos agrícolas e, consequentemente, sua conservação; a secagem, por sua vez, é o processo mais utilizado para assegurar a qualidade e a estabilidade dos grãos considerando-se que a diminuição da quantidade de água do material reduz a atividade biológica e as mudanças químicas e físicas que ocorrem durante o armazenamento.

O método de secagem intermitente, amplamente utilizado para a secagem do arroz em casca, é caracterizado pela passagem descontínua do ar aquecido pela massa de grãos em movimento, promovida pela recirculação do produto no secador. Com isto, a difusão da água do centro para a periferia do grão e a evaporação da água superficial se dão de maneira mais branda e equilibrada (Fagundes et al., 2005; Elias, 2007; Elias et al., 2010).

O secador é constituído de duas câmaras: uma de secagem, na qual ocorrem as trocas de energia e de massa durante o contato dos grãos com o ar aquecido e outra de equalização, em que os grãos permanecem na câmara, em repouso, sem que haja passagem de um fluxo de ar de secagem. Na câmara de secagem o ar cede energia térmica aos grãos, se resfria e absorve, na forma de vapor, a água periférica que evapora dos grãos de arroz. Na câmara de equalização o repouso do grão permite que a água mais interna do arroz migre para sua periferia, predominantemente por difusão (Elias et al., 2010).

Pelas características técnicas, operacionais e econômicas, o sistema intermitente é o mais recomendável para a secagem rápida do arroz devendo ser evitada a remoção brusca de água, que deve ser harmônica durante todo o processo, com temperatura do ar de secagem de no máximo $110^{\circ} \mathrm{C}$, para controlar os danos térmicos e mecânicos (Barbosa et al., 2005).

No estudo de sistemas de secagem, desenvolvimento e aperfeiçoamento de equipamentos, dimensionamento, otimização e determinação da viabilidade da aplicação comercial, são de fundamental importância a simulação e a obtenção de informações teóricas a respeito do comportamento de cada produto durante a remoção de água; em referência à simulação, utilizam-se modelos matemáticos que representam satisfatoriamente sua perda de água, durante a secagem.

Diversos modelos matemáticos têm sido utilizados para descrever o processo de secagem de produtos agrícolas. Embora várias teorias tenham sido propostas para predizer o comportamento da secagem de grãos e de sementes; na maioria das vezes, as relações semiempíricas e empíricas têm sido as melhores opções para descrever o processo de secagem apesar de sua validade estar restrita às condições sob as quais os dados experimentais foram obtidos (Brooker et al., 1992).

Comumente, os modelos se baseiam em variáveis externas em relação ao produto, como temperatura e umidade relativa do ar de secagem não fornecendo, entretanto, indicações sobre os fenômenos de transporte de energia e de água do interior dos grãos considerando-se, ainda, que todo o processo de secagem ocorre somente no período de taxa decrescente (Brooker et al., 1992).

Tem-se observados inúmeros trabalhos com o objetivo de identificar as características de diversos grãos e sementes durante a secagem como, por exemplo: feijão preto (Afonso Júnior \& Corrêa, 1999; Resende et al., 2007), café (Burmester \& Eggers, 2010); trigo parboilizado (Mohapatra \& Rao, 2005), arroz em casca (Shei \& Chen, 1999; Basunia \& Abe, 2001; Chen \&Wu, 2001; Cihan et al., 2007; Iguaz \& Vírseda, 2007), sementes de maracujá (Carlesso et al., 2005) e soja (Felipe \& Barrozo, 2003; Dondee et al., 2011) dentre outros. Considerando a importância do estudo teórico do processo de secagem dos produtos agrícolas e a limitação de informações teóricas a respeito de secagem intermitente de arroz em casca, desenvolveu-se este trabalho com o objetivo de ajustar diferentes modelos matemáticos aos valores experimentais em função do teor de água e estudar os efeitos das relações de intermitência na secagem intermitente de grãos de arroz na eficiência da operação.

\section{MATERIAL E MÉTODOS}

Foram utilizadas amostras de arroz irrigado produzidas na Região Sul do Rio Grande do Sul.

A colheita, mecanizada, foi realizada quando os grãos atingiram um teor de água próximo a $20 \%$ b.u; seguiu-se a operação de prelimpeza, sendo reduzidos os teores de impurezas e/ou matérias estranhas até valores próximos a $2 \%$.

No Laboratório de Pós-Colheita, Industrialização e Qualidade de Grãos da Faculdade de Agronomia Eliseu Maciel da Universidade Federal de Pelotas, RS, as amostras foram submetidas à secagem intermitente em um secador de fluxos cruzados sob diferentes condições de relação de intermitência entre a câmara de secagem e a de equalização, em três repetições:

- Relação de intermitência 1:1. Condição em que o secador foi carregado com $160 \mathrm{~kg}$ de arroz em $780 \mathrm{~s}$, ficando os grãos sob a ação do ar aquecido durante 380 s e equalizando por 380 s, em cada ciclo;

- Relação de intermitência 1:2. Nesta condição o secador foi carregado com $240 \mathrm{~kg}$ de arroz em 1.140 s, ficando os grãos sob a ação do ar aquecido por $380 \mathrm{~s}$ e equalizando durante $760 \mathrm{~s}$, em cada ciclo;

- Relação de intermitência 1:4. Nesta condição o secador foi carregado com $400 \mathrm{~kg}$ de arroz em $1.920 \mathrm{~s}$, ficando os grãos sob a ação do ar aquecido por $380 \mathrm{~s}$ e equalizando pelo tempo de $1.520 \mathrm{~s}$, em cada ciclo.

Durante todos os tratamentos de secagem foi mantido o mesmo manejo de temperatura do ar, sendo este crescente de $70 \pm 5^{\circ} \mathrm{C}, 90 \pm 5^{\circ} \mathrm{C}$ e $100 \pm 5^{\circ} \mathrm{C}$, respectivamente na $1^{\mathrm{a}}$ e na $2^{\mathrm{a}} \mathrm{e}$ da $3^{\text {a }}$ até a penúltima hora, havendo redução gradual da temperatura do ar, na última hora da operação.

O teor de água do produto foi determinado pelo método da estufa a $105 \pm 3{ }^{\circ} \mathrm{C}$, com circulação de ar durante $24 \mathrm{~h}$, utilizandose grãos não triturados (Brasil, 2009).

Durante os testes de secagem foram feitas, a cada hora, medições das temperaturas do ar ambiente (bulbo seco e de bulbo úmido), do ar de secagem, do ar de saída do secador (em dois pontos do revestimento, um no lado da entrada de calor e outro antes da entrada do ventilador) e da massa de grãos. A umidade relativa do ar ambiente e a umidade relativa do ar secante foram calculadas a partir de equações psicrométricas. 
Com vista à determinação da razão do teor de água do arroz em casca para as diferentes condições de secagem, utilizou-se aEq.1:

$$
\mathrm{RX}=\frac{\mathrm{X}-\mathrm{Xe}}{\mathrm{X}-\mathrm{Ue}}
$$

em que:

RX - razão do teor de água, adimensional

$\mathrm{X}$ - teor de água do arroz, decimal b.s

$\mathrm{Xi}$ - teor de água inicial, decimal b.s

Xe - teor de água de equilíbrio, decimal b.s

O teor de água de equilíbrio higroscópico foi calculado pela Eq. 2 proposta por Henderson-Thompson, para grãos de arroz em casca.

$$
\mathrm{XE}=0,01\left(\frac{\ln (1-\mathrm{UR})}{0,000019187(\mathrm{~T}+51,161)}\right)^{0,409}
$$

em que:

$\mathrm{XE}$ - umidade de equilíbrio, decimal b.s

$\mathrm{T}$ - temperatura dos grãos, ${ }^{\circ} \mathrm{C}$

UR - umidade relativa do ar no espaço intersticial, decimal

Aos dados experimentais de secagem intermitente de arroz em casca foram ajustados os modelos matemáticos mais utilizados para representação da secagem de produtos agrícolas (Afonso Júnior \& Corrêa, 1999; Akpinar et al., 2003; Ertekin \& Yaldiz, 2004; Lahsasni et al., 2004; Barbosa et al., 2007; Radünz et al., 2010, 2011; Reis et al., 2011), cujas expressões estão apresentadas na Tabela 1.

Para o ajuste dos modelos matemáticos foi realizada análise de regressão não-linear, pelo método Simplex e Quasi-Newton, sendo os valores dos parâmetros dos modelos estimados em função da temperatura do ar de secagem.

O ajuste do modelo aos dados experimentais foi avaliado em função da magnitude do coeficiente de determinação ajustado $\left(\mathrm{R}_{\text {ajustado }}\right)$, do erro médio relativo $(\mathrm{P})$ e do erro médio estimado (SE) e da verificação da falta de ajuste do modelo matemático, pela análise dos resíduos e dos gráficos de correspondência entre os valores estimados e os observados; erro médio relativo e o erro médio estimado para cada um dos modelos foram calculados conforme as Eqs. 3 e 4, respectivamente:

$$
\begin{aligned}
& P=\frac{100}{n} \sum_{i-1}^{n} \frac{[Y-Y o]}{Y} \\
& S E=\sqrt{\frac{\sum_{i-1}^{n}(Y-Y o)^{2}}{G L R}}
\end{aligned}
$$

em que:

Y - valor observado experimentalmente

Yo - valor calculado pelo modelo

$\mathrm{N}$ - número de observações experimentais
GLR - graus de liberdade do modelo (número de observações

\begin{tabular}{|c|c|c|}
\hline Modelo & Equação & \\
\hline Midilli & $R U=\operatorname{aexp}\left(-k \cdot t^{n}\right)+b \cdot t$ & (5) \\
\hline Page & $R U=\exp \left(-k \cdot t^{n}\right)$ & (6) \\
\hline Newton & $R U=\exp (-k \cdot t)$ & (7) \\
\hline $\begin{array}{l}\text { Exponencial de dois } \\
\text { termos }\end{array}$ & $R U=a \cdot \exp (-k \cdot t)+(1-a) \exp (-k \cdot a \cdot t)$ & (8) \\
\hline Dois termos & $R U=a \cdot \exp \left(-k_{0} \cdot t\right)+b \cdot \exp \left(-k_{1} \cdot t\right)$ & (9) \\
\hline Henderson \& Pabis & $R U=a \cdot \exp (-k \cdot t)$ & (10) \\
\hline $\begin{array}{l}\text { Henderson \& Pabis } \\
\text { modificado }\end{array}$ & $R U=a \cdot \exp (-k \cdot t)+b \cdot \exp \left(-k_{0} \cdot t\right)+c \cdot \exp \left(-k_{1} \cdot t\right)$ & $(11)$ \\
\hline Aproximação da difusão & $R U=a \cdot \exp (-k \cdot t)+(1-a) \cdot \exp (-k \cdot b \cdot t)$ & (12) \\
\hline Wang $\&$ Singh & $R U=1+(a \cdot t)+\left(b \cdot t^{2}\right)$ & (13) \\
\hline Verna & $R U=a \cdot \exp (-k \cdot t)+(1-a) \exp \left(-k_{1} \cdot t\right)$ & (14) \\
\hline Logarítmico & $R U=a \cdot \exp (-k \cdot t)+c$ & (15) \\
\hline Logistic & $R U=a_{0} / 1 \cdot a \cdot \exp (k \cdot t)$ & (16) \\
\hline
\end{tabular}
menos o número de parâmetros do modelo)

Tabela 1. Modelos matemáticos utilizados para predizer o fenômeno de secagem de produtos agrícolas

em que:

$\mathrm{k}, \mathrm{k}_{0}, \mathrm{k}_{1}$ - constantes de secagem, $\mathrm{s}^{-1}$

a, ao, b, c, n - coeficientes dos modelos

\section{RESULTADOS E DISCUSSÃO}

Na Figura 1 se encontram os valores experimentais da secagem intermitente de arroz realizada nas diferentes relações de intermitência, entre a câmara de secagem e de equalização.

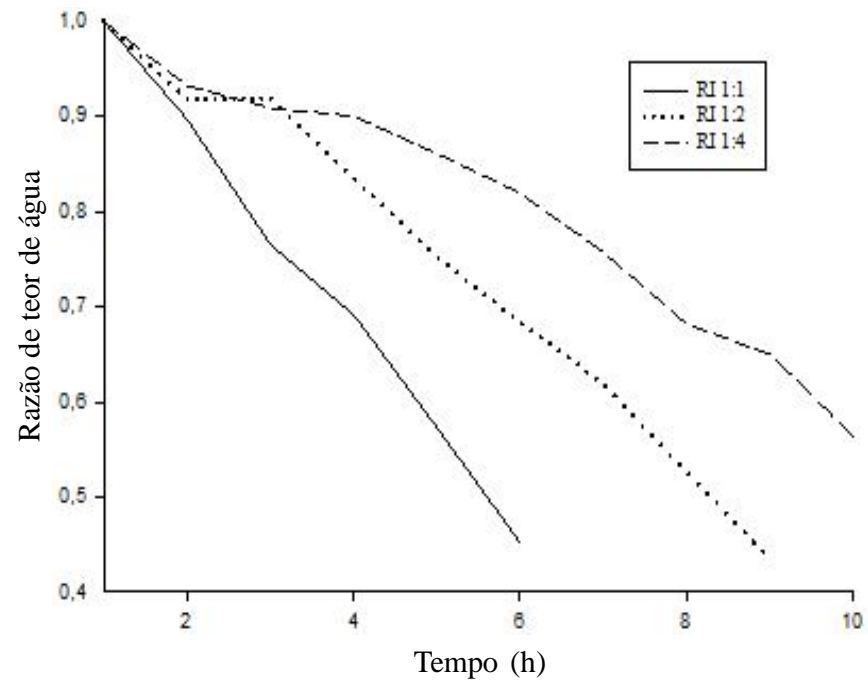

Figura 1. Valores experimentais da razão de teor de água e do tempo de secagem para três relações de intermitência $1: 1,1: 2,1: 4$, durante a secagem intermitente de arroz

Analisando a Figura 1 verifica-se que o tempo necessário para o arroz atingir o teor de água de aproximadamente $13 \%$, foi de 5, 8 e $10 \mathrm{~h}$ para as relações de intermitência de 1:1, 1:2 e 1:4, respectivamente. Com a diminuição da relação de intermitência ocorre maior taxa de remoção de água do produto evidenciando o aumento da taxa de secagem, fato observado por Barbosa et al. (2005) e Lacerda Filho et al. (2006). 
$\mathrm{Na}$ Figura 2 são plotadas as curvas que representam as temperaturas da massa de grãos de arroz, no decorrer das secagens, resultantes das médias dos valores obtidos entre as três repetições, para cada relação de intermitência.

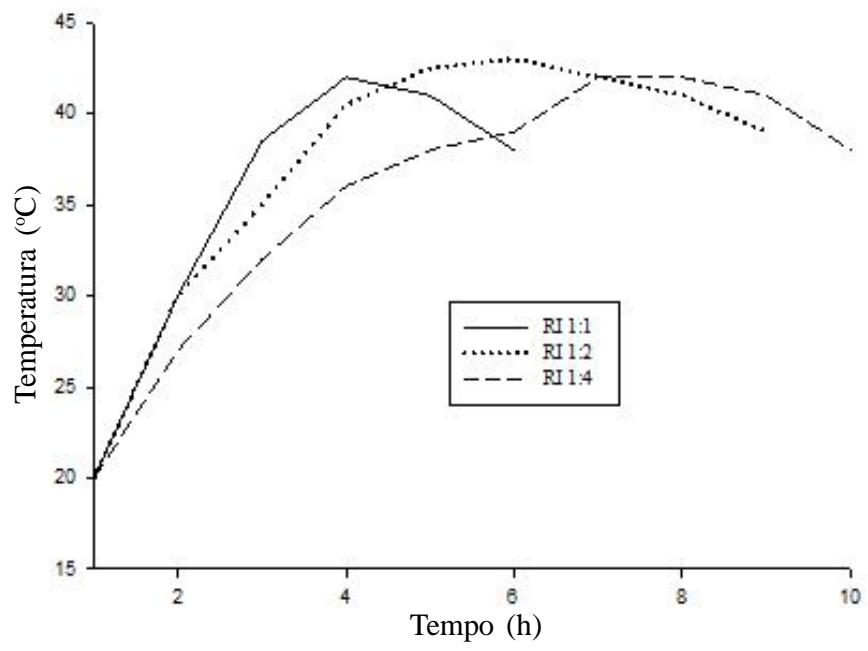

Figura 2. Temperatura da massa de grãos nas operações de secagens com RI 1:1, 1:2 e 1:4

Nota-se que as três curvas são semelhantes e que a temperatura da massa não ultrapassou $43{ }^{\circ} \mathrm{C}$, em todos os tratamentos. Isto demonstra que o manejo térmico dos grãos de arroz na operação de secagem foi adequado (Barbosa et al., 2006).

Em todos os tratamentos as temperaturas da massa dos grãos crescem mais nas duas primeiras horas, tendendo à estabilização, uma vez que o processo de secagem é composto de duas etapas hidrotérmicas distintas e complementares que incluem a difusão da água do interior do grão para a periferia e a evaporação da água periférica, sendo a primeira endotérmica e a segunda isoentálpica, quando ocorrem os fenômenos de transferência de calor e massa, em acordo com os princípios da termodinâmica.

A troca de calor por vapor de água ocorre na zona de secagem levando ao aquecimento dos grãos e ao resfriamento do ar de saída. Sempre que a água dos grãos vai diminuindo no interior do produto, a troca de energia em forma de calor por água decresce e, consequentemente, a temperatura da massa de grãos aumenta (Bonazzi et al., 1997).

A temperatura da massa de grãos é o parâmetro operacional intimamente ligado aos danos térmicos que podem ocorrer nos grãos em função da operação de secagem e/ou com o choque térmico, sendo esses responsáveis pelos danos imediatos e latentes cujos resultados se expressam mais em trincamentos e incidência de defeitos nos grãos de arroz, respectivamente.

A Figura 3 representa as taxas horárias de secagem dos grãos no decorrer da operação, resultantes das médias dos valores obtidos entre as três repetições, para cada relação de intermitência.

Observa-se, na Figura 3, que a taxa horária de secagem no tratamento com relação de intermitência 1:1, foi sempre superior à dos outros tratamentos. Os valores nesta operação variaram entre 1,35 e 1,85 pontos percentuais horários $\left(\mathrm{pp} \mathrm{h}^{-1}\right)$ mas não

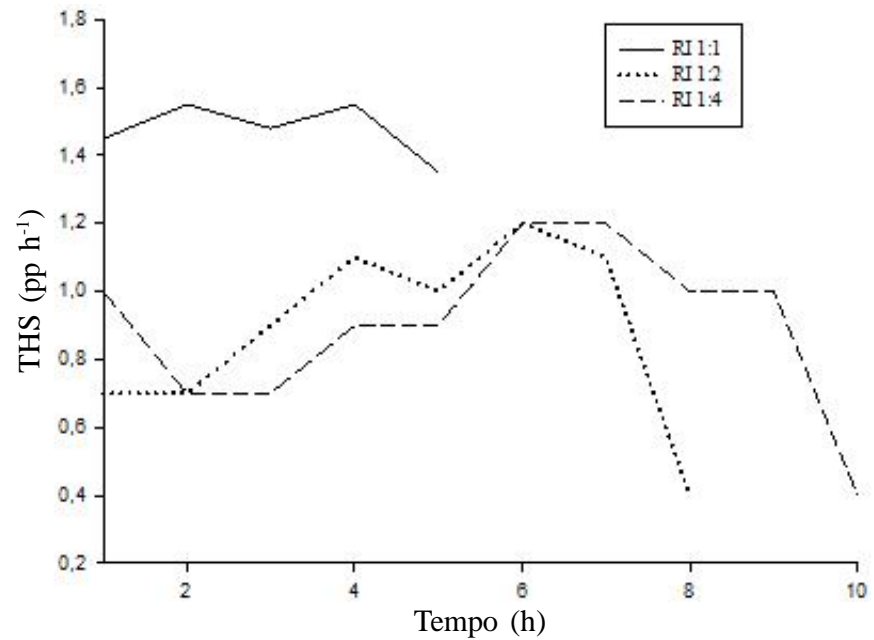

Figura 3. Taxa horária de secagem (TH S) nas operações de secagens com relações de intermitência 1:1, 1:2 e 1:4

ultrapassaram os $2 \mathrm{pp} \mathrm{h}^{-1}$ recomendados para a secagem do arroz em casca (Elias, 2007; Leite \& Vilella, 2010).

Examinando os comportamentos exibidos no gráfico, é possível notar que a taxa horária de secagem nos tratamentos 1:2 e 1:4 tem comportamento semelhante, com valores de até 1 $\mathrm{pp} \mathrm{h}^{-1}$ nas primeiras horas de secagem e máximo $1,2 \mathrm{pp} \mathrm{h}^{-1}$ entre a sexta e sétima hora de operação, fato este explicado pelo motivo dos grãos reterem energia até este intervalo de operação (sexta e sétima hora) quando ocorreram as maiores taxas de remoção de umidade, para esses dois tratamentos.

$\mathrm{Na}$ Tabela 2 se encontram os valores dos coeficientes de determinação ajustados, erros médios relativos e estimados e a tendência da distribuição dos resíduos para os modelos analisados, para a secagem intermitente de arroz, nas três condições de relação de intermitência.

Verificou-se, para os nove tratamentos de secagem de arroz, que os modelos matemáticos ajustados aos dados experimentais apresentaram coeficientes de determinação $\left(\mathrm{R}_{\text {ajustado }}^{2}\right)$ superiores ou próximos a 0,98 indicando, de acordo com Madamba et al. (1996), uma representação satisfatória do processo de secagem, exceto para os modelos de Newton, Exponencial de Dois Termos, Henderson e Pabis, Verna e Logistic. Para a maioria das condições analisadas os modelos de Midilli, Page, Dois termos, Henderson \& Pabis modificados, Aproximação da difusão, Wangh \& Sing e Logarítmico, apresentaram menores valores do erro médio estimado (SE) e magnitudes do erro médio relativo $(\mathrm{P})$ inferior a $10 \%$ sinalizando, de acordo com Mohapatra \& Rao (2005) serem adequados para descrição do processo. Verifica-se ainda que apenas o modelo de Midilli apresentou distribuição aleatória de resíduos (Figura 3 ) resultando, assim, em melhores ajustes aos dados experimentais de secagem intermitente de arroz, independente da relação de intermitência analisada.

Nas Figuras 4A, 4B e 4C são apresentadas as tendências de distribuição dos resíduos para os dados do modelo de Midilli, em função dos valores estimados da razão de umidade do arroz; os resíduos foram obtidos por meio da diferença entre os valores experimentais e os valores estimados pelo modelo. 
Tabela 2. Coeficientes de determinação ajustados $\left(\mathrm{R}^{2}{ }_{\text {aiustado }} \%\right)$, erros médio estimados (SE, decimal), erros médio relativos $(P, \%)$ e tendência de distribuição dos resíduos (Res), dos doze modelos analisados, para a secagem intermitente de arroz nas três condições de relação de intermitência

\begin{tabular}{|c|c|c|c|c|c|}
\hline $\begin{array}{l}\text { Relação de } \\
\text { intermitência }\end{array}$ & $\begin{array}{c}\text { Modelos } \\
\text { matemáticos }\end{array}$ & $\mathbf{R}_{\text {ajustado }}^{2}$ & SE & $\mathbf{P}$ & Res \\
\hline \multirow{12}{*}{$1: 1$} & Midilli & 99,84 & 0,0146 & 1,04 & Al \\
\hline & Page & 99,69 & 0,0356 & 1,94 & Al \\
\hline & Newton & 99,00 & 0,0637 & 3,25 & $\mathrm{Te}$ \\
\hline & Exponencial de dois termos & 98,98 & 0,0644 & 3,27 & $\mathrm{Te}$ \\
\hline & Dois termos & 99,18 & 0,0335 & 3,09 & $\mathrm{Te}$ \\
\hline & Henderson \& Pabis & 99,18 & 0,0579 & 3,09 & $\mathrm{Te}$ \\
\hline & Henderson $\&$ Pabis modificado & 99,18 & 0,0259 & 3,09 & $\mathrm{Te}$ \\
\hline & Aproximação da difusão & 99,72 & 0,0240 & 1,80 & $\mathrm{Al}$ \\
\hline & Wang $\&$ Singh & 99,83 & 0,0263 & 1,20 & $\mathrm{Te}$ \\
\hline & Verna & 99,78 & 0,0240 & 1,80 & $\mathrm{Al}$ \\
\hline & Logarítmico & 99,83 & 0,0188 & 1,32 & $\mathrm{Te}$ \\
\hline & Logistic & 99,18 & 0,0409 & 3,09 & $\mathrm{Te}$ \\
\hline \multirow{12}{*}{$1: 2$} & Midilli & 99,68 & 0,0249 & 1,28 & $\mathrm{Al}$ \\
\hline & Page & 99,44 & 0,0576 & 1,78 & $\mathrm{Te}$ \\
\hline & Newton & 96,97 & 0,1331 & 5,48 & $\mathrm{Te}$ \\
\hline & Exponencial de dois termos & 99,37 & 0,0609 & 2,07 & $\mathrm{Te}$ \\
\hline & Dois termos & 97,60 & 0,0685 & 5,38 & $\mathrm{Te}$ \\
\hline & Henderson \& Pabis & 97,60 & 0,1187 & 5,38 & $\mathrm{Te}$ \\
\hline & Henderson $\&$ Pabis modificado & 97,60 & 0,0530 & 5,38 & $\mathrm{Te}$ \\
\hline & Aproximação da difusão & 99,41 & 0,0417 & 1,98 & $\mathrm{Te}$ \\
\hline & Wang $\&$ Singh & 99,67 & 0,0442 & 1,21 & Al \\
\hline & Verna & 99,41 & 0,0417 & 1,98 & $\mathrm{Te}$ \\
\hline & Logarítmico & 99,17 & 0,0495 & 2,90 & $\mathrm{Te}$ \\
\hline & Logistic & 97,60 & 0,0839 & 5,38 & $\mathrm{Te}$ \\
\hline \multirow{12}{*}{ 1:4 } & Midilli & 99,61 & 0,0276 & 1,78 & $\mathrm{Al}$ \\
\hline & Page & 98,50 & 0,0933 & 2,97 & $\mathrm{Al}$ \\
\hline & Newton & 94,83 & 0,1717 & 6,05 & $\mathrm{Te}$ \\
\hline & Exponencial de dois termos & 94,83 & 0,1717 & 6,05 & $\mathrm{Te}$ \\
\hline & Dois termos & 99,65 & 0,0261 & 1,54 & $\mathrm{Al}$ \\
\hline & Henderson $\&$ Pabis & 95,53 & 0,1600 & 5,98 & $\mathrm{Te}$ \\
\hline & Henderson $\&$ Pabis modificado & 98,51 & 0,0415 & 3,25 & $\mathrm{Te}$ \\
\hline & Aproximação da difusão & 98,25 & 0,0713 & 3,36 & $\mathrm{Te}$ \\
\hline & Wang \& Singh & 99,27 & 0,0653 & 2,12 & $\mathrm{Te}$ \\
\hline & Verna & 94,83 & 0,1214 & 6,05 & $\mathrm{Te}$ \\
\hline & Logarítmico & 97,57 & 0,0839 & 4,45 & $\mathrm{Te}$ \\
\hline & Logistic & 95,53 & 0,1131 & 5,98 & $\mathrm{Te}$ \\
\hline
\end{tabular}

Al - Aleatório; Te - Tendencioso

Na Figura 5 são apresentadas as curvas de secagem elaboradas com os dados experimentais e os valores simulados pela equação de Midilli, para a secagem intermitente de arroz em casca.

Na 5 observa-se elevada correlação entre os valores experimentais e os estimados e um comportamento satisfatório da distribuição de resíduos gerados por essas equações (Figuras 4A, 4B e 4C), aproximando-se das disribuições normais esperadas. O modelo de Midilli mostrou-se mais apropriado para predição do fenômeno de secagem intermitente de grãos de arroz por apresentar os menores desvios e os melhores ajustes.

Os resultados encontrados neste trabalho mostram que a equação de Midilli foi a que apresentou melhor ajuste aos dados experimentais de secagem, também observado por Cihan et al. (2006) para a secagem de arroz em casca.
A.
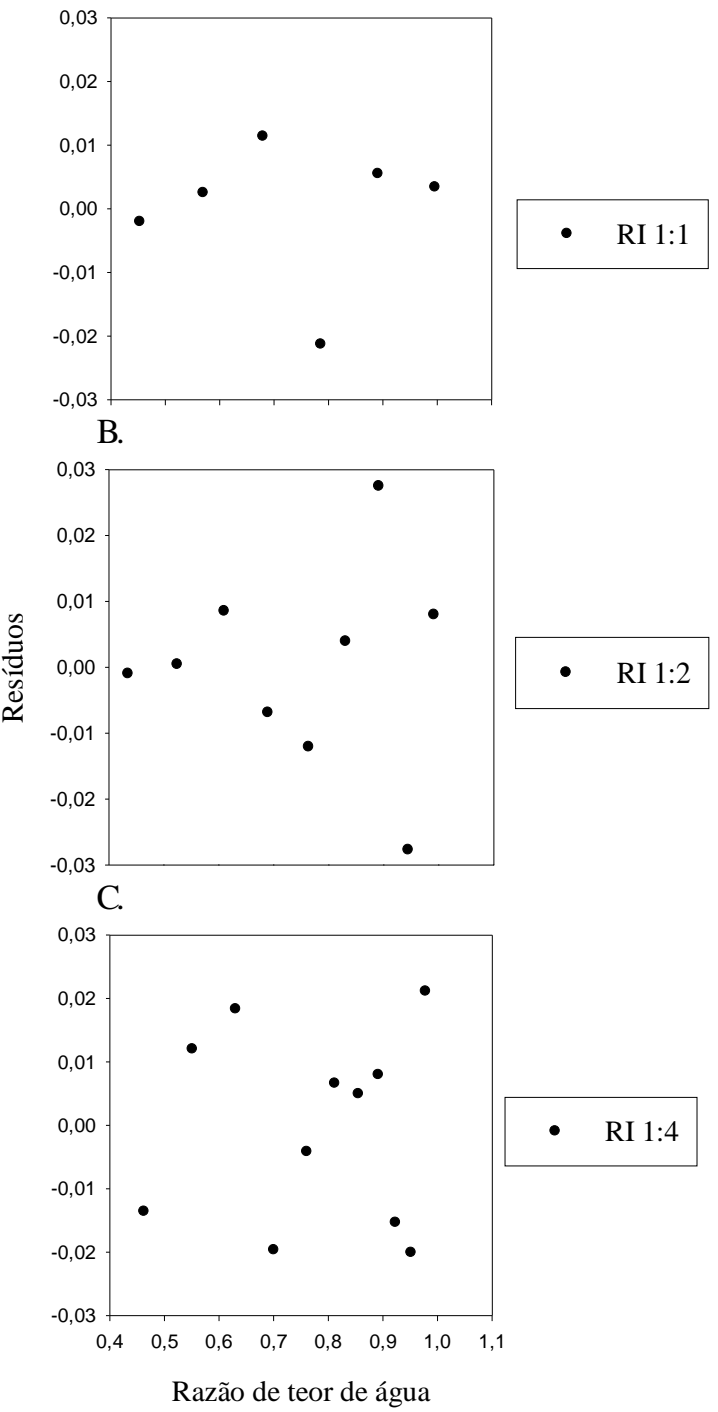

Figura 4. Tendência de distribuição dos resíduos para o modelo de Midilli durante a secagem intermitente de arroz nas relações de intermitência de 1:1 (A), 1:2 (B) e 1:4 (C) em função dos valores estimados da razão de teor de água

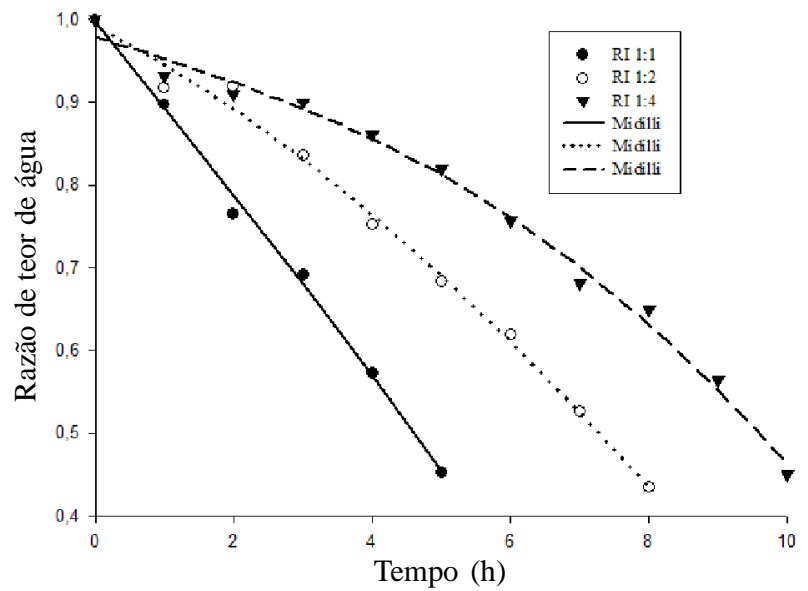

Figura 5. Valores experimentais da razão de teor de água (RX) de grãos de arroz em casca calculados pela equação de M idilli, para as relações de intermitência de 1:1, 1:2 e $1: 4$ 


\section{ConclusõEs}

1. Para um mesmo manejo de temperatura o aumento da relação de intermitência entre as câmaras de secagem e de equalização, aumenta o tempo de secagem.

2. A temperatura da massa de grãos não é independente da relação de intermitência e, sim, do manejo operacional de secagem.

3. A relação de intermitência interfere na taxa horária de secagem dos grãos durante a operação.

4. A equação de camada delgada proposta por Midilli é a que melhor representa a secagem intermitente de arroz em casca, independente da relação de intermitência.

\section{LITERATURA CITADA}

Afonso Júnior, P. C.; Corrêa, P. C. Comparação de modelos matemáticos para descrição da cinética de secagem em camada fina de sementes de feijão. Revista Brasileira de Engenharia Agrícola e Ambiental, v.3, p.349-353, 1999.

Akpinar, E. K; Bicer, Y.; Yildiz, C. Thin layer drying of red pepper. Journal of Food Engineering, v.59, p.99-104, 2003.

Barbosa, F. da F.; Elias, M. C.; Fagundes, C. A. A.; Pereira, F. M.; Radünz, L. L. Efeitos das secagens estacionária e intermitente e do tempo de armazenamento no desempenho industrial de grãos de arroz. Revista Brasileira de Armazenamento, v.30, p.83-90, 2005.

Barbosa, F. da F.; Melo, E. de C.; Santos, R. H. S.; Rocha, R. P. da; Martinazzo, A. P.; Radünz, L. L.; Gracia, L. M. N. Evaluation of mathematical models for prediction of thinlayer drying of brazilian lemon-scented verbena leaves (Lippia alba (Mill) N. E. Brown). Revista Brasileira de Produtos Agroindustriais, v.9, p.71-80, 2007.

Barbosa, F. da F.; Milmann, M. J. M.; Fagundes, C. A.; Martins, I. G.; Schownke, O. N.; Cunha Neto, A. C.; Peter, M. Z.; Elias, M. C. Effect of stationary and intermittent drying on latent damages in rice grains stored. In: International Working Conference on Stored Product Protection, 9, 2006, Campinas. Proceedings...Campinas: Abrapós, 2006. p.1003-1011.

Basunia, M. A.; Abe, T. Moisture desorption isotherms of medium grain rough rice. Journal of Stored Products Research, v.37, p.205-219, 2001.

Bonazzi, C.; Dupeuty, M. A.; Hermelin, A. Influence of drying conditions on the processing quality of rough rice. Drying Technology, v.15, p.1141-1157, 1997.

Brasil. Ministério da Agricultura e Reforma Agrária. Regras para análise de sementes. Brasília: DNDV/CLAV, 2009. 365p.

Brooker, D. B.; Bakker-Arkema, F. W.; Hall, C. W. Drying and storage of grains and oilseeds. Westport: The AVI Publishing Company, 1992. 450p.

Burmester, K; Eggers R. Heat and mass transfer during the coffee drying process. Journal of Food Engineering, v.99, p.430-436, 2010.

Carlesso, V. O.; Berbert, P. A.; Silva, R. F.; Vianna, A. P.; Detmann, E.; Dionello, R. G. Secagem de sementes de maracujá em camada delgada. Revista Brasileira de Fruticultura, v.27, p.444-448, 2005.

Chen, C.; Wu, P. C. Thin-layer drying model for rough rice with high moisture content. Journal of Agriculture Engineering Research, v.80, p.45-52, 2001.
Cihan, A.; Kahveci, K.; Hacihafizoglu, O. Modelling of intermittent drying of thin layer rough rice. Journal of Food Engineering, v.79, p.293-298, 2007.

Dondee, S.; Meeso, N.; Soponronnarit, S.; Siriamornpun, S. Reducing cracking and breakage of soybean grains under combined near-infrared radiation and ûuidized-bed drying. Journal of Food Engineering, v.104, p.6-13, 2011.

Elias, M. C. Pós-colheita de arroz: Secagem, armazenamento e qualidade. 3.ed. Pelotas: UFPel, 2007. 437p.

Elias, M. C.; Oliveira, M.; Schiavon, R. A. Qualidade de arroz na pós-colheita: Ciência, tecnologia e normas. Pelotas: Santa Cruz, 2010. 543p.

Ertekin, C.; Yaldiz, O. Drying of eggplant and selection of a suitable thin layer drying model. Journal of Food Engineering, v.63, p.349-359, 2004.

Fagundes, C. A. A.; Elias, M. C.; Barbosa, F. F. Desempenho industrial de arroz secado com ar aquecido por queima de lenha e de GLP. Revista Brasileira de Armazenamento, v.30, p.8-15, 2005.

Felipe, C. A. S.; Barrozo, M. A. S. Drying of soybean seeds in a concurrent moving bed: Heat and mass transfer and quality analysis. Drying Technology, v.21, p.439- 456, 2003.

Iguaz, A.; Vírseda, P. Moisture desorption isotherms of rough rice at high temperatures. Journal of Food Engineering, v.79, p.794-802, 2007.

Lacerda Filho, A. F.; Ferreira, A. 1. B.; Silva, J. S.; Ramos, A. M. Secagem de arroz parboilizado em secador de fluxos concorrentes. Revista Brasileira de Armazenamento, v.31, p.170-180, 2006.

Lahsasni, S.; Kouhila, M.; Mahrouz, M.; Jaouhari, J. T. Drying kinetcs of prickly pear fruit (Opuntia fícus indica). Journal of Food Engineering, v.61, p.173-179, 2004.

Leite, C. W.; Villela, A. F. Velocidade de secagem de arroz em secador intermitente rápido com o emprego de dois fluxos de ar. Informativo ABRATES, v.20, p.010-014, 2010.

Madamba, P. S.; Driscoll, R. H.; Buckle, K. A. Thin layer drying characteristics of garlic slices. Journal of Food Engineering, v.29, p.75-97, 1996.

Mohapatra, D.; Rao, P. S. A thin layer drying model of parboiled wheat. Journal of Food Engineering, v.66, p. 513-518, 2005.

Radünz, L. L.; Amaral, A. S.; Mossi, A. J.; Melo, E. C.; Rocha, R. P. Avaliação da cinética de secagem de carqueja. Engenharia na Agricultura, v.19, p.19-27, 2011.

Radünz, L. L.; Mossi, A. J.; Zakrzevski, C. A.; Amaral, A. S.; Grassmann, L. Análise da cinética de secagem de folhas de sálvia. Revista Brasileira de Engenharia Agrícola e Ambiental, v.14, p.979-986, 2010.

Reis, R. C.; Barbosa, L. S.; Lima, M. L.; Reis, J. S.; Devilla, I. A.; Ascheri, D. P. R. Modelagem matemática da secagem da pimenta Cumari do Pará. Revista Brasileira de Engenharia Agrícola e Ambiental, v.15, p.347-353, 2011.

Resende, O.; Corrêa, P. C.; Jaren, C.; Moure, A. J. Bean moisture diffusivity and drying kinetics: a comparison of the liquid diffusion model when taking into account and neglecting grain shrinkage. Spanish Journal of Agricultural Research, v.5, p.51-58, 2007.

Shei, H. J.; Chen, Y. L. Thin layer models for intermittent drying of rough rice. Journal of Food Engineering, v.76, p.577-581, 1999. 